\title{
Wave transport through thin slabs of random media with internal reflection: Ballistic to diffusive transition
}

\author{
Xiangdong Zhang and Zhao-Qing Zhang* \\ Department of Physics and Institute of Nano Science and Technology (INST), The Hong Kong University of Science and Technology, \\ Clear Water Bay, Kowloon, Hong Kong, China \\ (Received 15 March 2002; published 26 July 2002)
}

\begin{abstract}
The static and dynamic properties of wave transport through thin slabs of random media in the presence of internal reflection are investigated by performing first-principles calculations. These results are compared with results from time-independent and time-dependent diffusion equations, respectively, where the effects due to internal reflection are incorporated into an average extrapolation length in the boundary conditions. For the static properties, we find an abrupt transition from ballistic to diffusive behavior when sample thickness is about three mean free paths, i.e., $L \approx 3 l$. The diffusion approximation is valid when $L>3 l$, independent of the amount of internal reflection. For the dynamic properties, both the peak arrival time at short times and the diffusion constant at long times of the transmitted pulse indicate that there is a region of anomalous diffusion when $3 l<L<L_{c}$. The diffusion constant in this region increases with decreasing $L$. It also increases with the amount of internal reflection. The physical origin of the existence of such an anomalous region is the resonance-induced wave focusing effect. Due to the presence of internal reflection, the wave energy tends to concentrate in the forward direction at output boundary. It makes direction randomization difficult in the scattered waves. A similar wave focusing effect has been found in resonant tunneling systems of electrons in the presence of elastic scattering. The diffusion approximation is valid when $L>L_{c}$. The value of $L_{c}$ is about ten times the average extrapolation length, i.e., $L \approx 10 z_{e}$, where $z_{e}$ is a fast increasing function of the amount of internal reflection.
\end{abstract}

DOI: 10.1103/PhysRevE.66.016612

PACS number(s): 42.25.Bs, 42.25.Dd

\section{INTRODUCTION}

The study of wave propagation through random media has been an intensive field of research for many decades [1]. Many aspects of the transport of waves in random media, such as coherent backscattering, continuous-wave transmission, pulse propagation, and speckle correlations, are well described by the diffusion approximation [2-5]. Despite the success and widespread use of this simple approximation, limitations do exist. One such limitation occurs in thin samples, where the number of scatterings becomes insufficient to randomize the phases of the emerging waves and a crossover to ballistic transport must ultimately occur. In recent years, many works have discussed such crossover behavior [6-14]. In experimental respects, many investigations have probed such crossover behavior with different transition lengths, which depend on the physical quantities measured. For example, diffusing wave spectroscopy (DWS) experiments [6] and steady state photon transmission measurements [3] have indicated that the transport of photons is diffusive when sample thicknesses $L$ is as low as 3-5 transport mean free paths $l$. In contrast, pulsed optical transmission measurements [7-9] have reported systematic deviations from diffusion theory at much larger values of $L / l \leqslant 8-10$. Within the context of DWS, a solution of the transport equation has been derived to account for the contributions of short scattering paths, which are increasingly ballistic in na-

\footnotetext{
*Corresponding author. FAX: 852-2358-1652. Email address: phzzhang@ust.hk
}

ture, resulting in a simple correction to the diffusion approximation [10]. In another approach, the telegrapher equation with suitable boundary conditions has been employed to account for the role of ballistic transport and scattering anisotropy [11]. In a recent work [12], ultrasonic pulse transmission experiments in strongly scattering media consisting of glass beads immersed in water have been carried out. At the same time, first-principles calculations of both the frequency correlation function of the transmitted field and the timedomain profile of the transmitted intensity have been performed by solving the ladder approximation of the BetheSalpeter equation with the use of the bulk Green's function. From the peak arrival time, both theory and experiments have exhibited an abrupt crossover between ballistic and diffusive behaviors when $L / l \approx 3$.

Although the Bethe-Salpeter equation describes the transport of waves in thin samples where the diffusion approximation breaks down, the use of the bulk Green's function becomes inadequate when the system possesses large internal reflection at the sample boundaries. Internal reflection have been shown, both experimentally and theoretically, to be of considerable importance in the diffusive transport of light through random media [15-20]. In the presence of large internal reflection, a Green's function that includes the multiple reflections from the boundaries should be used. Such a study has been carried out on a semiinfinite sample [20]. However, a systematic first-principles study of wave transport through thin samples of random media in the presence of large internal reflection has not been conducted. For thick samples, an improved diffusion approximation has been successfully used in interpreting experimental results $[3,7]$. In this improved diffusion approximation, the effects 
of internal reflection at the boundaries is incorporated into a single extrapolation length $l_{e}$ in the mixed boundary condition $[15,16]$. It has been shown that this improved diffusion approximation becomes asymptotically exact in the limit of the large index mismatch in a semifinite sample [20]. However, the validity of the improved diffusion approximation in thin samples has not been tested.

In this work, we study wave transport through thin slabs of random media by performing first-principles calcuations using the ladder approximation for the Bethe-Salpeter equation, in which a renormalized single-particle averaged Green's function that incorporates all internal reflections from the boundaries will be used. Thus, our calculations go beyond the limitations of the diffusion approximation and allow us to include the coherent multiple reflections of waves from two boundaries. Thus, our results are valid for thin samples with any amount of internal reflection. Our focus is on the crossover behavior in the ballistic-to-diffusive transition as a function of the index mismatch from both static and dynamic points of view. For the static quantities, we study the total transmitted intensity under a plane-wave incident. For the dynamic quantities, we study the shape of timeresolved transmitted pulse under a pulsed plane-wave incident. For the static behavior, we find an abrupt transition from ballistic to diffusive behavior when $L / l \approx 3$. The diffusion approximation is valid when $L / l>3$, independent of the amount of internal reflection. However, for the dynamic properties, from both the peak arrival time at short times and the diffusion constant at long times of the transmitted pulse, we find a region of anomalous diffusion when $3 l<L<L_{c}$, in which the diffusion constant increases with decreasing $L$. The physical origin of the existence of such an anomalous region is the resonance-induced wave focusing effect. Due to the presence of internal reflection, the wave energy tends to concentrate in the forward direction. This focusing effect makes the randomization of wave propagation direction more difficult. A similar wave focusing effect has been found in resonant tunneling systems of electrons in the presence of elastic scattering [21]. The diffusion approximation is valid when $L>L_{c}$. The value of $L_{c}$ is about ten times the averaged extrapolation length, i.e., $L \approx 10 z_{e}$, where $z_{e}$ is a fast increasing function of the amount of internal reflection. In Sec. II, we present the analytical formulas for both the BetheSalpeter equation and the improved diffusion theory. The results of these calculations are presented in Sec. III. Section IV presents the discussion as well as the conclusions of this work.

\section{THEORY}

We consider a pulsed plane wave that is normally incident on the front surface, $z=0$, of a slab-shaped sample containing isotropic random scatterers. The thickness of the sample is $L$. The optical index is assumed to take different values in three regions, namely, $n_{1}$ outside the scattering medium $(z$ $<0), n$ inside the scattering medium $(0<z<L)$, and $n_{2}$ outside the scattering medium $(z>L)$. To investigate wave transport through this scattering medium, we are interested in modeling the temporal evolution of the intensity and the fre- quency correlation function of the field, probed at the back face of the sample at $z=L$. At any time $t$ and position $\vec{r}$, the field is specified by the time-dependent wave function, which can be written as

$$
\psi(t, \vec{r})=(2 \pi)^{-1} \int d \Omega \exp (-i \Omega t) f(\Omega) \phi_{\Omega}(\vec{r}),
$$

where $f(\Omega)$ describes the spectrum of frequencies $\Omega$, contained in the pulse and $\phi_{\Omega}(\vec{r})$ is the spatial part of the wave function. In order to obtain useful physical information, we have to consider the ensemble-averaged quantities for the random systems. The ensemble-averaged intensity corresponding to Eq. (1) can be expressed as

$$
\begin{aligned}
\left\langle|\psi(t, \vec{r})|^{2}\right\rangle= & (2 \pi)^{-2} \int d \Omega\left[\int d \omega f\left(\Omega^{+}\right) f^{*}\left(\Omega^{-}\right)\right. \\
& \left.\times\left\langle\phi_{\Omega^{+}}(\vec{r}) \phi_{\Omega^{-}}^{*}(\vec{r})\right\rangle \exp (-i \omega t)\right],
\end{aligned}
$$

where \langle\rangle denotes configurational averaging, and the frequencies $\Omega^{ \pm}=\Omega \pm(\omega / 2)$ have been written in terms of the center frequency $\Omega$ and the difference or modulation frequency $\omega$, the latter also being conjugate to the travel time $t$. From Eq. (2) it is clear that the ensemble-averaged intensity $\left\langle|\psi(t, \vec{r})|^{2}\right\rangle$ is given by the Fourier transform of the frequency correlation function $C_{\Omega}(\omega, \vec{r})=\left\langle\phi_{\Omega^{+}}(\vec{r}) \phi_{\Omega^{-}}^{*}(\vec{r})\right\rangle$, which is the fundamental quantity to be determined. $C_{\Omega}(\omega, \vec{r})$ can be obtained from the space-frequency correlation function $\widetilde{C}_{\Omega}\left(\omega, \vec{r}, \overrightarrow{r^{\prime}}\right)=\left\langle\phi_{\Omega^{+}}(\vec{r}) \phi_{\Omega^{-}}^{*}\left(\overrightarrow{r^{\prime}}\right)\right\rangle$, which is known to satisfy the Bethe-Salpeter equation

$$
\begin{aligned}
\widetilde{C}_{\Omega}\left(\omega, \vec{r}, \overrightarrow{r^{\prime}}\right)= & \left\langle\varphi_{i n c}\left(\vec{r}, \Omega^{+}\right)\right\rangle\left\langle\varphi_{i n c}^{*}\left(\overrightarrow{r^{\prime}}, \Omega^{-}\right)\right\rangle \\
& +\int d \overrightarrow{r_{1}} d \overrightarrow{r_{2}} d \overrightarrow{r_{3}} d \overrightarrow{r_{4}}\left\langle G_{\Omega^{+}}\left(\vec{r}, \vec{r}_{1}\right)\right\rangle \\
& \times\left\langle G_{\Omega^{-}}^{*}\left(\overrightarrow{r^{\prime}}, \overrightarrow{r_{2}}\right)\right\rangle U\left(\overrightarrow{r_{1}}, \overrightarrow{r_{3}} ; \overrightarrow{r_{2}}, \vec{r}_{4}\right) \widetilde{C}_{\Omega}\left(\omega, \overrightarrow{r_{3}}, \overrightarrow{r_{4}}\right),
\end{aligned}
$$

where $\left\langle\varphi_{\text {inc }}\left(\vec{r}, \Omega^{ \pm}\right)\right\rangle$is the configurationally averaged incident wave function inside the sample and takes the following forms for the case of a normally incident plane wave considered here [20]:

$$
\begin{gathered}
\left\langle\varphi_{i n c}(z, \Omega)\right\rangle=e^{i p_{1} z}+r e^{-i p_{1} z}, \quad z<0, \\
\left\langle\varphi_{i n c}(z, \Omega)\right\rangle=t_{1} e^{i \tilde{p z}}+t_{2} e^{-i \tilde{p} z}, \quad 0<z<L, \\
\left\langle\varphi_{i n c}(z, \Omega)\right\rangle=t e^{i p_{2} z}, \quad z>0,
\end{gathered}
$$

where $p_{1}=\Omega / v_{1}, \tilde{p}=\Omega / v+i /(2 l)$, and $p_{2}=\Omega / v_{2}$. Here $v_{1}=c / n_{1}, v=c / n$, and $v_{2}=c / n_{2}$ are the wave speeds in three different regions. For convenience, we set the value of $c$ to unity. The coefficients $r, t_{1}, t_{2}$, and $t$ should be determined from the boundary conditions at the two surfaces. It is easy to show that $t_{1}$ and $t_{2}$ take the forms: 


$$
\begin{aligned}
& t_{1}=\frac{2 p_{1}\left(\tilde{p}+p_{2}\right)}{\left(\tilde{p}+p_{1}\right)\left(\tilde{p}+p_{2}\right)-\left(\tilde{p}-p_{1}\right)\left(\tilde{p}-p_{2}\right) e^{2 i \tilde{p} L}}, \\
& t_{2}=\frac{2 p_{1}\left(\tilde{p}-p_{2}\right) e^{2 i \tilde{p} L}}{\left(\tilde{p}+p_{1}\right)\left(\tilde{p}+p_{2}\right)-\left(\tilde{p}-p_{1}\right)\left(\tilde{p}-p_{2}\right) e^{2 i \tilde{p} L}} .
\end{aligned}
$$

The coefficients $r$ and $t$ will not be needed in our calculations.

The function $U$ in Eq. (3) stands for the summation of all irreducible vertex functions. For isotropic scattering, the scattering mean free path is equal to the transport mean free path and they are both denoted by $l$. The lowest-order contribution to the vertex function becomes [22]

$$
U\left(\overrightarrow{r_{1}}, \overrightarrow{r_{3}}, \overrightarrow{r_{2}}, \overrightarrow{r_{4}}\right)=\frac{4 \pi}{l} \delta\left(\overrightarrow{r_{1}}-\overrightarrow{r_{3}}\right) \delta\left(\overrightarrow{r_{1}}-\overrightarrow{r_{2}}\right) \delta\left(\overrightarrow{r_{3}}-\overrightarrow{r_{4}}\right)
$$

With this form for $U$, Eq. (3) generates a sum of ladder diagrams, which represents the multiple scattering of waves without interference effects. The averaged single-particle Green's function $\left\langle G_{\Omega}\left(\vec{r}, \vec{r}^{\prime}\right)\right\rangle$ in Eq. (3) represents the coherent wave transport inside the sample between two scatterings. In the presence of internal reflection, the function $\left\langle G_{\Omega}\left(\vec{r}, \vec{r}^{\prime}\right)\right\rangle$ loses its translational invariance in the $z$ direction and possesses the translational invariance only in the transverse plane. It is more convenient to write $\left\langle G_{\Omega}\left(\vec{r}, \overrightarrow{r^{\prime}}\right)\right\rangle$ as

$$
\left\langle G_{\Omega}\left(\vec{r}, \vec{r}^{\prime}\right)\right\rangle=(2 \pi)^{-1} \int\left\langle G\left(z, z^{\prime} ; q\right)\right\rangle \exp \left[i \vec{q} \cdot\left(\vec{\rho}-\overrightarrow{\rho^{\prime}}\right)\right] d \vec{q},
$$

where $\vec{\rho}$ denotes the position of $\vec{r}$ in the transverse plane. $\vec{q}$ is the transverse wave vector. In order to incorporate all the multiple reflections between two surfaces, the function $\left\langle G\left(z, z^{\prime} ; q\right)\right\rangle$ must satisfy the following one-dimensional wave equation [20]:

$$
\begin{gathered}
\left(\frac{d^{2}}{d z^{2}}-q^{2}+k_{1}^{2}\right)\left\langle G\left(z, z^{\prime} ; q\right)\right\rangle=0, \quad z<0, \\
{\left[\frac{d^{2}}{d z^{2}}-q^{2}+\left(k+\frac{i}{2 l}\right)^{2}\right]\left\langle G\left(z, z^{\prime} ; q\right)\right\rangle=0, \quad 0<z, \quad z^{\prime}<L,} \\
\left(\frac{d^{2}}{d z^{2}}-q^{2}+k_{2}^{2}\right)\left\langle G\left(z, z^{\prime} ; q\right)\right\rangle=0, \quad z>0 .
\end{gathered}
$$

From the above equations, the Green's functions corresponding to the three regions can be obtained easily. In our calculations, we need only the Green's function inside the slab region $(0<z<L)$, which takes the form:

$$
\begin{aligned}
G\left(z, z^{\prime} ; q\right)= & \frac{1}{2 i p}\left[\frac{1}{r_{1} r_{2}-e^{-2 i L p}}\right] \\
& \times\left[r_{1} e^{-2 i d p} e^{i p\left(z+z^{\prime}\right)}+e^{-2 i L p} e^{i p\left|z-z^{\prime}\right|}\right. \\
& \left.+r_{1} r_{2} e^{-i p\left|z-z^{\prime}\right|}+r_{2} e^{-i p\left(z+z^{\prime}\right)}\right],
\end{aligned}
$$

where $r_{1}=\left(p-p_{0}\right) /\left(p+p_{0}\right), r_{2}=\left(p-p_{0}^{\prime}\right) /\left(p+p_{0}^{\prime}\right)$ and $p_{0}^{2}$ $=k_{1}^{2}-q^{2} \geqslant 0, p^{2}=(k+i / 2 l)^{2}-q^{2}$, and $p_{0}^{\prime 2}=k_{2}^{2}-q^{2} \geqslant 0$.

Since, for our purpose, only the frequency correlation function $C_{\Omega}(\omega, \vec{r})$ is needed, we can solve the BetheSalpeter equation for the simpler case where $\vec{r}^{\prime}=\vec{r}$. Thus, Eq. (3) becomes

$$
\begin{aligned}
\widetilde{C}_{\Omega}(\omega, z)= & \left\langle\varphi_{\text {inc }}\left(\vec{r}, \Omega^{+}\right)\right\rangle\left\langle\varphi_{\text {inc }}^{*}\left(\vec{r}, \Omega^{-}\right)\right\rangle \\
& +\frac{2}{l} \int d z_{1} H\left(z, z_{1}\right) \times \widetilde{C}_{\Omega}\left(\omega, z_{1}\right),
\end{aligned}
$$

where

$$
H\left(z, z_{1}\right)=\int_{0}^{\infty} q G_{\Omega^{+}}\left(z, z_{1}, q\right) G_{\Omega^{-}}^{*}\left(z, z_{1}, q\right) d q .
$$

Equation (11) takes the form of the Milne equation. It is a Fredholm integral equation of the second kind with a singular kernel that can be solved numerically by using the standard method [22,23]. After the frequency correlation function is obtained, it is straightforward to calculate the temporal variation of the transmitted intensity from Eq. (2). In our calculations, we focus on a particular center frequency $\Omega$. Thus, the precise form of $f(\Omega)$ is irrelevant. Since we ignore all the interference effects in the Bethe-Salpeter equation, our results are valid when $n \Omega l \gg 1$. In this limit, our results are insensitive to the value of $\Omega$.

For the static quantity, we calculate the diffusive part of the total transmitted intensity at the output surface, which can be obtained from the quantity $\widetilde{C}_{\Omega}(0, \vec{L}, \vec{L})$ $-\left\langle\varphi_{\text {inc }}(\vec{L}, \Omega)\right\rangle\left\langle\varphi_{\text {inc }}^{*}(\vec{L}, \Omega)\right\rangle$ in Eq. (2). In order to compare the results obtained from the above first-principles calculations with those of the diffusion approximation, we summarize below some known analytical results for the diffusion approximation. The time-dependent diffusion equation for the intensity in a slab of thickness $L$ takes the form:

$$
\frac{\partial I(\vec{r}, t)}{\partial t}-D_{o} \nabla^{2} I(\vec{r}, t)+I(\vec{r}, t)=Q(\vec{r}, t),
$$

where $D_{o}=l / 3 n$ is the bulk diffusion constant of the scattering medium. $Q(\vec{r}, t)$ denotes the source function. For a pulsed plane-wave incident, we can write $Q$ as $\delta\left(z-z_{p}\right)$ $\delta(t)$. Here $z_{p}$ represents the coherent penetration depth of the source. For a plane-wave incident, the intensity becomes a function of $z$ and $t$. As for the boundary conditions, it has been shown that the effects of internal reflection can be accounted for by incorporating an extrapolation length $z_{e}$ into the boundary conditions of the diffusion equation, which is related to the internal reflection through 


$$
z_{e}=\operatorname{al}(1+R) /(1-R),
$$

where $a=2 / 3$ and $R$ represents the ratio of the incoming flux to the outgoing flux and can be obtained from the angleaveraged reflection coefficient, i.e., $R=\langle R(\theta)\rangle$ [16]. For scalar waves, the formula for $\langle R(\theta)\rangle$ is given in Table I of Ref. [20]. In the absence of internal reflection, the transport theory gives the Miline result of $a=0.71$ [24]. If both boundaries have the same index mismatch, the solution of Eq. (13) takes the form [15]:

$$
\begin{aligned}
I(z, t)= & \frac{2}{d} \sum_{m=1}^{\infty} \sin \left[\frac{m \pi}{d}\left(z_{e}+z_{p}\right)\right] \sin \left[\frac{m \pi}{d}\left(z+z_{e}\right)\right] \\
& \times \exp \left[-D_{o} t\left(\frac{m \pi}{d}\right)^{2}\right],
\end{aligned}
$$

where $d=L+2 z_{e}$. In the static limit of a continuous planewave incident, the source function becomes $Q=\delta\left(z-z_{p}\right)$. The static solution of Eq. (13) gives the following intensity at $z=L$ :

$$
I(L)=\frac{3 z_{e}\left(z_{e}+z_{p}\right)}{l v\left(2 z_{e}+L\right)} .
$$

Equation (16) shows that the inverse of $I(L)$ is a straight line. The zero of $I(L)^{-1}$ is twice the extrapolation length $z_{e}$. In fact, from the solutions of the Bethe-Salpeter equation at large sample thicknesses, one can also obtain numerically the value of $z_{e}$ from Eq. (16) by extrapolating $I(L)$ to negative $L$. This result should be compared with the prediction of Eq. (14) used in the diffusion approximation.

Finally, we determine the penetration depth $z_{p}$, used in the diffusion approximation in the following way. We first assume a distribution of $z_{p}$ weighted by an exponential attenuation of the coherent intensity [25]. Since $I(L)$ is linear in $z_{p}$, the average intensity, $\langle I(L)\rangle_{z_{p}}$ takes the form of Eq. (16), except replacing $z_{p}$ by an average penetration depth, $\left\langle z_{p}\right\rangle$ [25], i.e.,

$$
\left\langle z_{p}\right\rangle=\frac{\int_{0}^{L / l} z_{p} e^{\left(-z_{p} / l\right)} d\left(z_{p} / l\right)}{\int_{0}^{L / l} e^{\left(-z_{p} / l\right)} d\left(z_{p} / l\right)} .
$$

It is easy to see that when $L \gg l,\left\langle z_{p}\right\rangle \approx l$.

\section{NUMERICAL RESULTS}

For simplicity, we first consider the case of a slab of scattering medium with an optical index $n$, embedded in a homogeneous background with optical index unity. Thus, both surfaces of the slab have the same index mismatch. The cases in which two boundaries have different mismatches will be considered later. We numerically solve Eqs. (2) and (3) at different values of $n$ and $L / l$. For the static case, the diffusive part of the transmitted intensity at the output surface is obtained from the numerical solution of Eq. (2) through $I(L)=\widetilde{C}_{\Omega}(0, \vec{L}, \vec{L})-\left\langle\varphi_{\text {inc }}(\vec{L}, \Omega)\right\rangle\left\langle\varphi_{\text {inc }}^{*}(\vec{L}, \Omega)\right\rangle$. We plot the inverse of $I(L)$ as a function of $L / l$ in Figs. $1(\mathrm{a}-\mathrm{c})$ in circles for $n=1,1.3$, and 1.6, respectively. The case of $n=1$ represents no internal reflection. It is interesting to see that all these three cases show similar crossover behavior. For $L / l>3$, the data points follow three straight lines, indicating diffusive behavior according to Eq. (16). The extrapolation length $z_{e}$ can readily be obtained by extrapolating these lines to the real axes. We find $z_{e} / l \approx 0.7,1.7$, and 3.2 for $n=1,1.3$, and 1.6, respectively. These numbers agree excellently with the prediction of Eq. (14) when $a=0.71$ is used. If we use the calculated values of the angle-averaged reflection coefficient $R=0.43$ and 0.65 for $n=1.3$ and 1.6, respectively, according to Eq. (14), we find $z_{e} / l \approx 1.78$ and 3.35. The values of $z_{e} / l$ obtained here will be used in the dynamic calculations below. Figure 1 clearly shows an abrupt crossover from ballistic to diffusive behavior at a transition thickness of $L / l \approx 3$, independent of the index mismatch. Thus, for the static behavior, the improved diffusion approximation of Eq. (16) is valid as long as $L / l>3$, which is insensitive to the amount of internal reflection at the sample boundaries. This conclusion is consistent with the experimental data given in Ref. [3].

For the dynamical behavior, the time-resolved transmitted intensities $I(L, t)$, calculated from Eqs. (2) and (3) for a slab of $L / l=8$ with $n=1,1.3$, and 1.6 are shown as dotted curves in Figs. 2(a-c), respectively. Here, for convenience, we have normalized the intensity so that its peak value is unity. The three insets show the semilog plots of the same quantities. A straight line at the long intervals is expected from the diffusion approximation. Its slope gives the decay time, which in turn determines the diffusion constant through $1 / \tau_{D}$ $=\pi^{2} D(L) /\left(L+2 z_{e}\right)^{2}$ [7]. In Fig. 2, we also plot the results of Eq. (15) as solid lines in each case for comparison. For the case of $n=1$, the excellent agreement between the results of Eqs. (2), (3), and (16) in Fig. 2(a) indicates that the behavior of the pulse propagation through a slab of thickness $L / l=8$ can be well described by the diffusion approximation. However, this is not true for the cases of $n=1.3$ and $n=1.6$. A discernible deviation appears when $n=1.3$. This derivation becomes more distinct as the value of $n$ is increased. In particular, we notice that the slopes of the dotted lines in the insets of Figs. 2(b) and 2(c) are different from those of the solid lines. This indicates diffusive behavior but with a diffusion constant different from that of the bulk system, i.e., $D(L) \neq D_{o}=l / 3 n$. This anomalous transport behavior in thin samples has been reported in various experiments $[7,12,21]$. Thus, for the dynamic behavior, unlike in the static case, there exists an anomalous diffusive region between the ballistic transport and the diffusive transport of a bulk system in which a scale-dependent diffusion constant appears. To understand better the transport behavior in this anomalous region, we study systematically both the peak arrival time $t_{p}$ at short times and the diffusion constant $D(L)$ at long times. The $\log -\log$ plot of $t_{p}$ as a function of $L / l$ at $n=1,1.3$, and 1.6 are shown as open circles in Figs. 3(a-c), respectively. The solid lines are the results obtained from Eq. (15). The result of Fig. 3(a) for the case of $n=1$ recovers the previous 


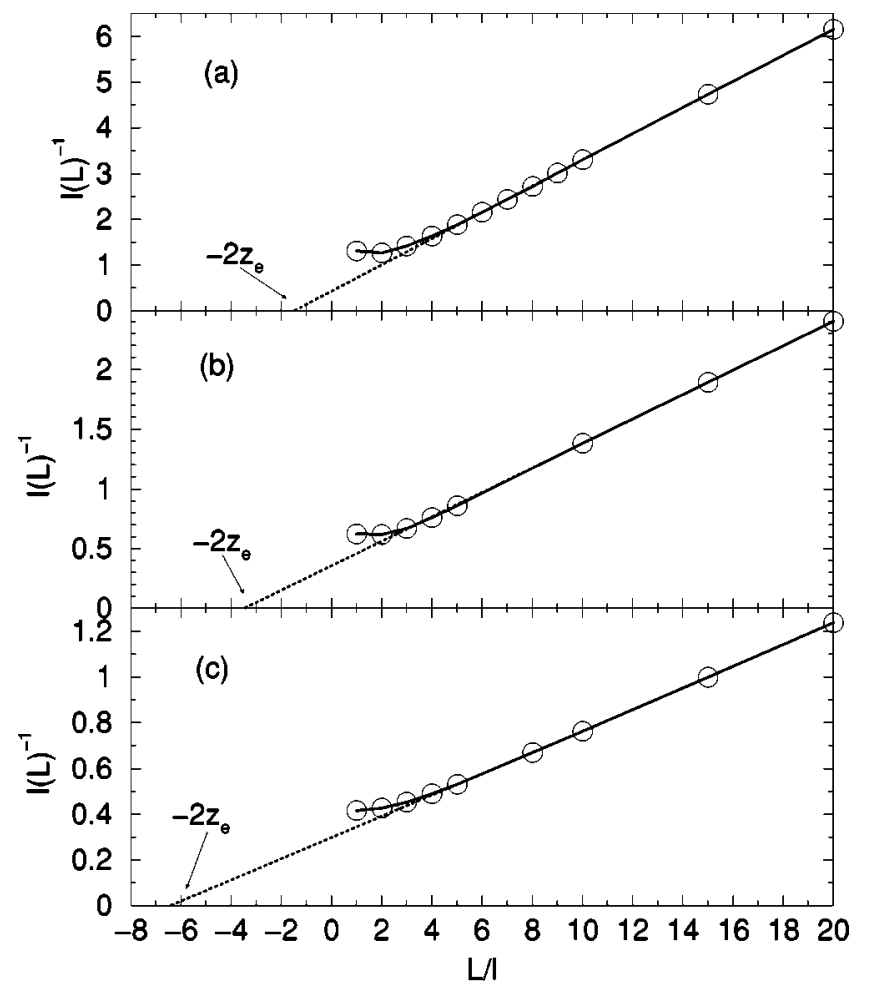

FIG. 1. The inverse of intensity $I(L)^{-1}$, as a function of the slab thickness for $n=1$ (a), $n=1.3$ (b), and $n=1.6$ (c). The solid lines are the calculated results and the dotted lines are the fitted results.

result of Ref. [12], where a bulk Green's function was used in Eq. (3). The two-segment behavior shown in Fig. 3(a) clearly indicates a rather abrupt crossover from ballistic to diffusive behavior at $L / l=3$, below which the slope is 1 and above which it changes to 2 suddenly. Such an abrupt transition was first reported in Ref. [12]. However, if we look more carefully, there exists a slight difference between the solid line and circles (marked by the dotted line) in the region of $3 \geqslant L / l \geqslant 5$. This difference, as well as the region it spans, increases with the amount of internal reflection as can be seen from Figs. 3(b) and 3(c). For the cases of $n=1.3$ and 1.6 , the circles overlap with the solid lines when $L / l \approx 15$ and 30 , respectively. These values are about ten times the extrapolation length in each case. The existence of this anomalous region becomes more obvious in Fig. 4, where the normalized diffusion constant $D(L) / D_{o}$ is plotted as a function of $L / l$ for $n=1$ (diamonds), $n=1.3$ (squares), and $n=1.6$ (circles). The deviations from unity represent the anomalous diffusion. It first appears when $L / l \approx 8,15$, and 32 for $n$ $=1,1.3$, and 1.6, respectively. These results are consistent with the analyses of $t_{p}$ shown in Fig. 3. From Fig. 4, we also find that $D(L)$ increases with $n$, but decreases with $L$. The physical reason for such a behavior will be discussed below. Thus, for the dynamic behavior, we can separate the wave transport into three different regions: (i) ballistic, (ii) anomalous diffusive, and (iii) bulk diffusive. The crossover thickness is very sensitive to the amount of internal reflection.

Finally, we consider the case in which two surfaces have different index mismatches. For convenience, we consider the experimental systems given in Ref. [7], in which the

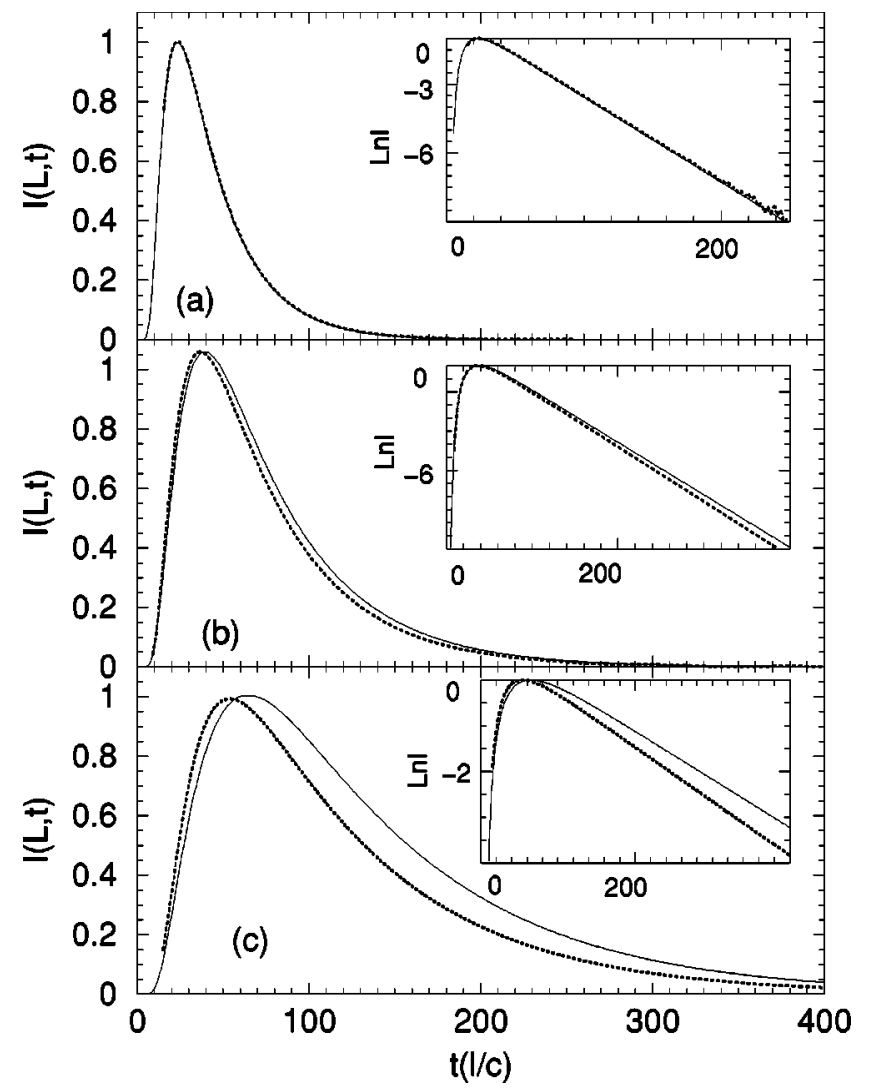

FIG. 2. The time profile of the transmitted intensity for the sample thickness $L / l=8$ with different index mismatches. (a) $n$ $=1$, (b) $n=1.3$, and (c) $n=1.6$. The solid lines are the results from the diffusion theory [Eq. (15)] and the dotted lines are from Eq. (2). The dot-dashed line marks the ballistic segment.

values of $n_{1}, n$, and $n_{2}$ are, respectively, 1, 1.34, and 1.46. In this case, the angle-averaged reflection coefficients are $R_{l}$ $=0.47$ and $R_{r}=0.02$ for the left and right boundaries, respectively. The corresponding extrapolation lengths are $1.97 l$ and $0.74 l$, yielding an average value of $z_{e}=1.36 l$. The result of $D(L) / D_{o}$ is plotted as triangles in Fig. 4. Again, deviation from the diffusion approximation appears when $L \approx 10 l$, which is about $7 z_{e}-8 z_{e}$.

\section{DISCUSSION AND CONCLUSIONS}

In order to understand the physical origin of the anomalous diffusion in thin samples, we plot in Fig. 5(a) the scattered static intensity $I(z)=\widetilde{C}_{\Omega}(0, \vec{z}, \vec{z})$ $-\left\langle\varphi_{\text {inc }}(\vec{z}, \Omega)\right\rangle\left\langle\varphi_{\text {inc }}^{*}(\vec{z}, \Omega)\right\rangle$ as a function of $z$ for the case of $L / l=2$ and $n=1.6$ at $\Omega=16$ (solid curve) and $\Omega=8$ (dotted curve). The existence of large spatial oscillations in $I(z)$ indicates that the scattered waves possess a significant portion of the coherent property. It is also seen that the oscillations become more significant near the sample boundaries due to the presence of internal reflection. The number of oscillations inside the sample is roughly equal to $n L \Omega / \pi$, which has the value 8.14 (16.3) when $\Omega=8(16)$. For the sake of comparison, in Fig. 5(b), we also plot the coherent part of the 


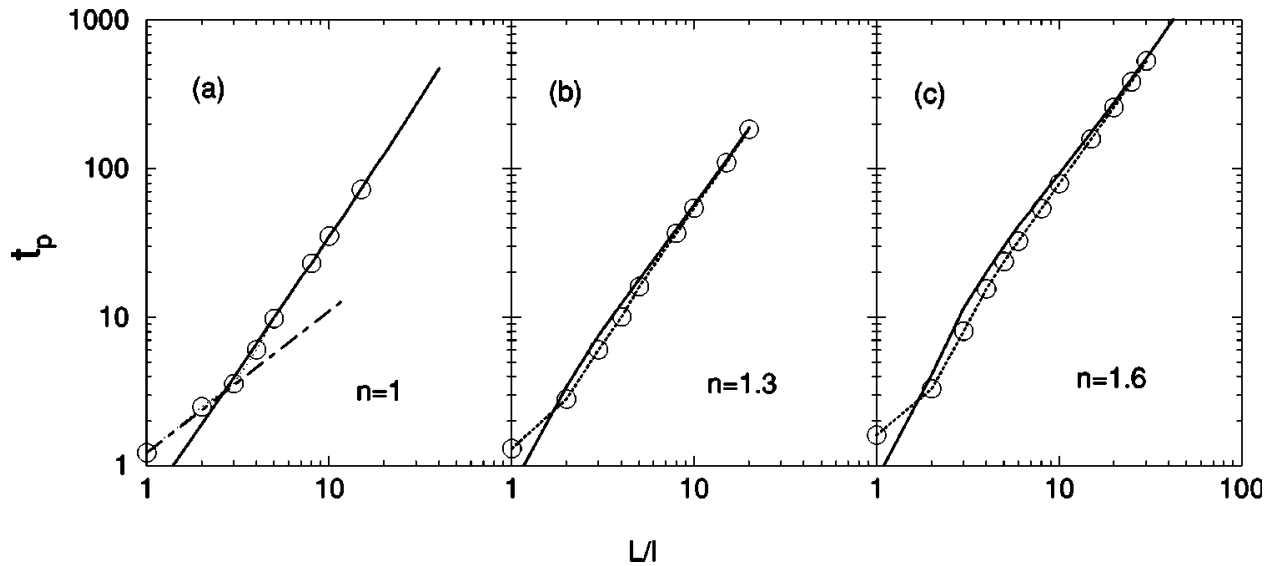

FIG. 3. The peak positions in the time profile of the transmitted intensity plotted as a function of the sample thickness, $L / l$. (a) $n$ $=1$, (b) $n=1.3$, and (c) $n=1.6$. The solid lines are the results from the diffusion theory [Eq.(15)] and the dotted lines are from Eq. (2).

wave, i.e., $\left\langle\varphi_{i n c}(\vec{z}, \Omega)\right\rangle\left\langle\varphi_{i n c}^{*}(\vec{z}, \Omega)\right\rangle$. Although the number of oscillations between the coherent and scattered parts are the same, there is a major difference between the two. The coherent intensity has an exponential decay, whereas the scattered intensity decays linearly. A linear decay is the characteristics of diffusive transport. Thus, the scattered waves show both ballistic and diffusive behavior. This is not surprising for such a thin sample, i.e., $L / l=2$. When the sample thickness is increased, it is expected that such oscillations will die off and a full diffusive behavior will be reached. In Fig. 6, we plot the similar curves for the case of $L / l=4$. It is indeed found that overall oscillations have reduced significantly. However, large oscillations still persist near sample boundaries where the period approximately equals to $\pi / n \Omega$. The presence of such enhanced oscillations is entirely due to internal reflection and has significant effects on the dynamical transport. Since the period of these oscillations is close to that of the coherent wave, it suggests that the wave energy tends to concentrate in a resonant state that has small transverse wave vector. A similar wave focusing phenomenon has

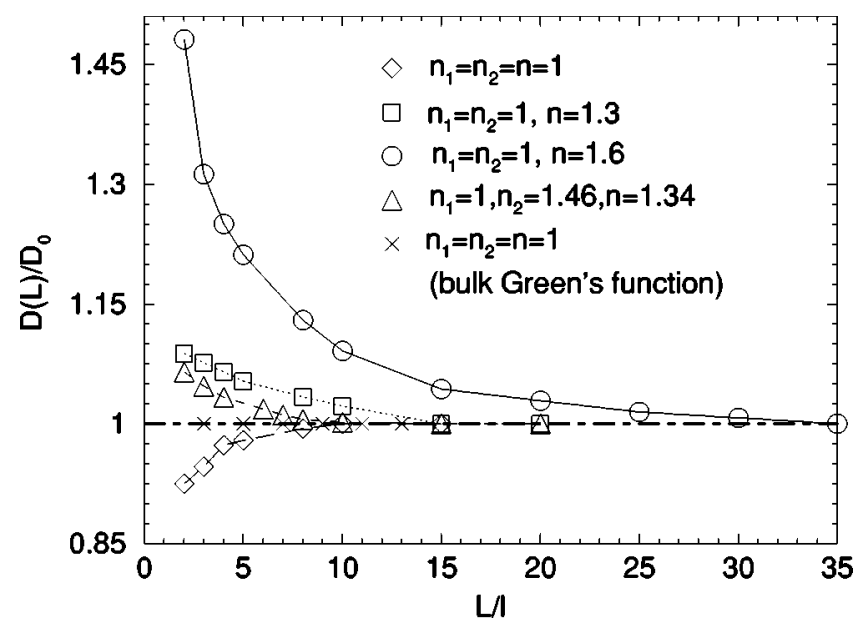

FIG. 4. The calculated diffusion constants as a function of sample thickness $L$. The bulk diffusion constant is characterized by the diffusion constant $D_{0}$. The plotted data are scaled with $D_{0}$ (dotdashed line). The diamonds are for $n=1$, the squares for $n=1.3$, the circles for $n=1.6$, and triangles for experimental results [7]. Crosses represent the results calculated using the bulk Green's function. been observed for electrons in resonant tunneling systems [21]. The presence of elastic scattering tends to focus the tunneling electrons into a resonant state. Thus, the multiple reflection of the waves between two boundaries tends to lock the wave scattering into the forward direction and make the direction randomization difficult. Due to this wave focusing effect, the incident angles of the scattered waves at the sample boundaries are not evenly distributed. Thus, the angle-averaged reflection coefficient calculated according to an even distribution of incident angles actually overestimates the value of $R$ [16]. This, in turn, overestimates the extrapolation length $l_{e}$, and therefore, gives a larger value of $D(L) / D_{o}$. The spatial oscillations of $I(z)$ die off when $L$

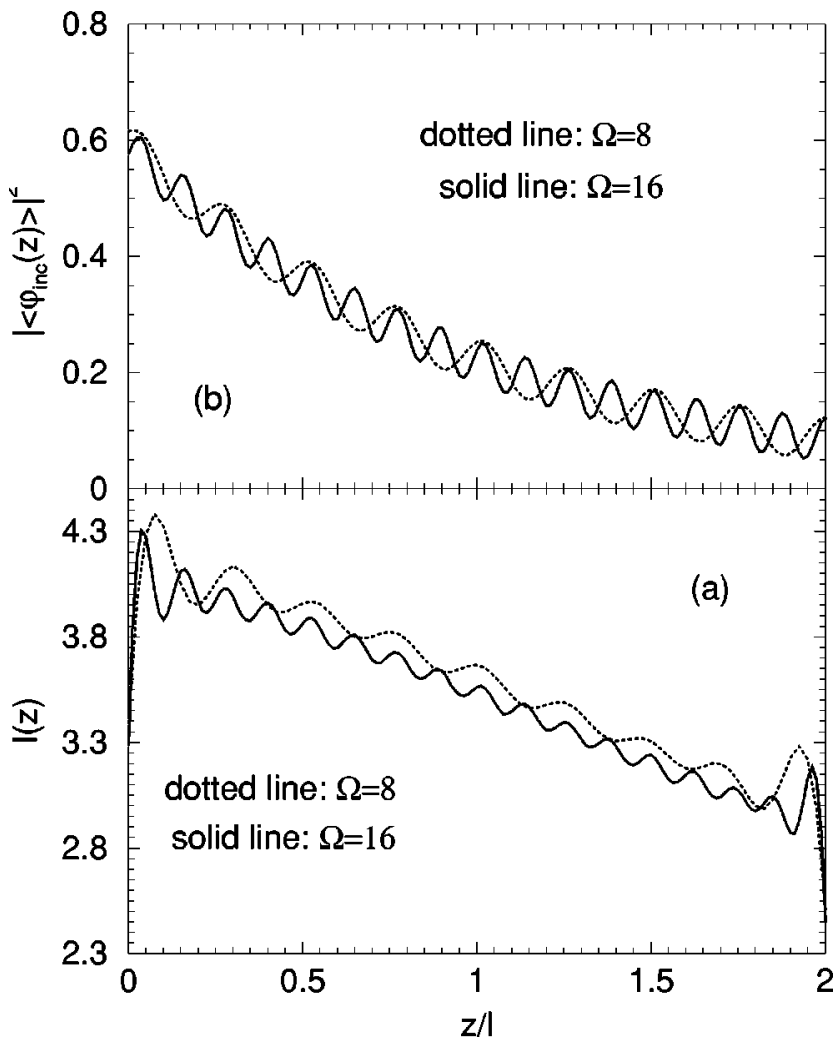

FIG. 5. (a) The scattered intensity $I(z)$ as a function of $z / l$ for $L / l=2$ slab. The dotted line is for $\Omega=8$ and the solid line for $\Omega$ $=16$. Figure $5(\mathrm{~b})$ represents the coherent wave intensity. 


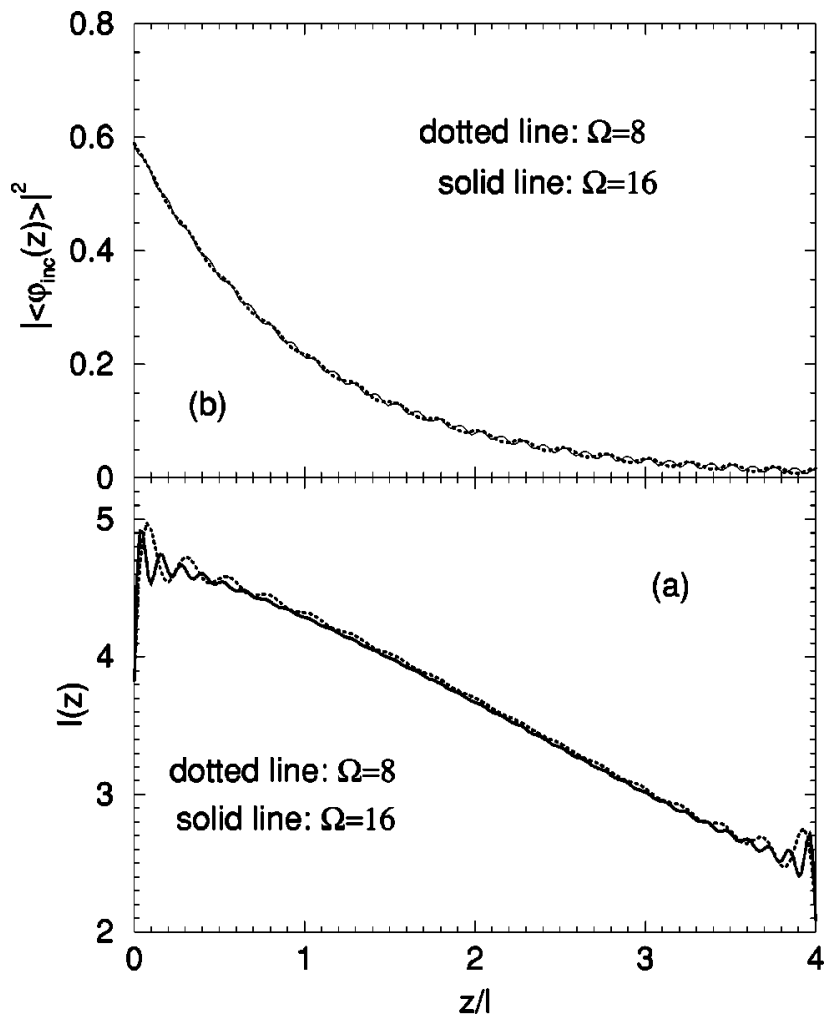

FIG. 6. Same as Fig. 5(a) except $L / l=4$.

becomes large or when index mismatch becomes small. They disappear entirely when sample thickness is about $10 z_{e}$. This explains why $D(L) / D_{o}$ shown in Fig. 4 increases with increasing $n$ and with decreasing $L$.

It is also interesting to notice in Fig. 5(a) that the scattered intensities $I(z)$, at $z=0$ and $z=L$ are independent of $\Omega$. In fact, this is also true when $\omega \neq 0$. In other words, the function $\widetilde{C}_{\Omega}(\omega, \vec{L}, \vec{L})-\left\langle\varphi_{i n c}\left(\vec{L}, \Omega^{+}\right)\right\rangle\left\langle\varphi_{i n c}^{*}\left(\vec{L}, \Omega^{-}\right)\right\rangle$is insensitive to the value of $\Omega$ when $n \Omega l \gg 1$. Thus, our previous results shown in Figs. 1-4 are independent of the value of $\Omega$ used as long as $\Omega$ lies in the weak scattering regime, i.e., $n \Omega l$ $\gg 1$. To show this, we plot in Fig. 7, the time-resolved transmitted intensity $I(L, t)$, calculated from both $\Omega=8$ (open circles) and $\Omega=16$ (filled squares) for the case of $L / l=2$. The two curves completely overlap each other.

For the case of $n=1$, our Eqs. (2) and (3) underestimate the diffusion constant in thin samples, i.e., $D(L) / D_{o} \leqslant 1$. The reason is as follows. Even when $n=1$, a small amount of internal reflection still exists at the boundaries due to the presence of disorder inside the sample, as can be seen from the term $i / 2 l$ in Eq. (9). This small amount of internal reflection has been included in Eq. (2) through the use of $\left\langle\varphi_{\text {inc }}\right\rangle$ and $G$ in Eqs. (4) and (9), respectively. However, it is not included in the calculation of $z_{e}$ in Eq. (14). Thus, such an underestimation of $z_{e}$ produces a smaller $D(L)$. In fact, when we use the bulk Green's function and the function $\varphi_{\text {inc }}$ used in Ref. [12], the results of $D(L) / D_{o}$ are shown as crosses in Fig. 4. In this case, the Bethe-Salpeter equation excellently reproduces the results of the diffusion approximation for $L \geqslant 3$.

Finally, we should point out again that, in this work, we

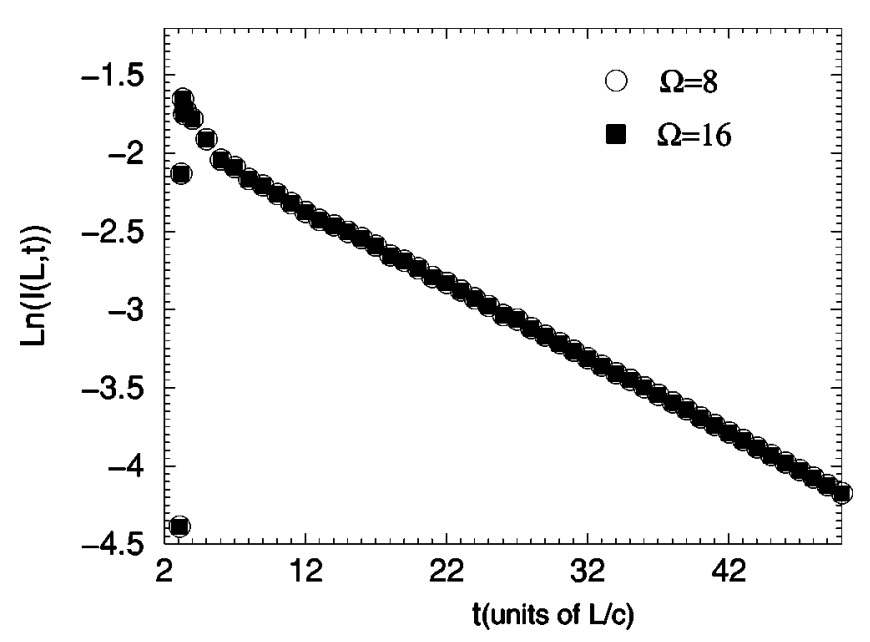

FIG. 7. The time-resolved transmitted intensity, $\operatorname{Ln}[I(L, z)]$, as a function of $t$ (in units of $L / c$ ) for $L / l=2 \mathrm{slab}$. The open circles represent $\Omega=8$ and the filled squares are for $\Omega=16$.

consider only the weak scattering regime, where $n \Omega l \gg 1$, and no interference effects have been included in our calculations. However, the experimental observations of anomalous transport in strongly scattering media always show a reduction of $D(L)$ in thin samples $[7,12,25]$. The reduction may be due to wave interference effects. In thin samples, the intensity at long times come from waves that have long travel paths in the transverse plane. A reduced dimensionality in thin samples likely makes interference important. How to incorporate the effects due to interference into the diffusion constant in thin samples with internal reflection remains an interesting and challenging task for future study.

In conclusion, the static and dynamic features of wave transport through a slab of strong scattering medium in the presence of internal reflection is investigated by performing first-principle calculations. Our calculations, based on the ladder approximation of the Bethe-Salpeter equation with the use of a renormalized Green's function, are valid in the weak scattering regime for any amount of internal reflection. They can be applied to any waves satisfying the classical wave equation. The effects of internal reflection on the transition from ballistic to diffusive behavior are analyzed in detail. An abrupt crossover from ballistic to diffusive transition has been found when $L \approx 3 l$. However, from the analyses of the dynamic properties, we find a region of anomalous diffusion when $3 l<L<L_{c}$, in which the diffusion constant increases with decreasing $L$. The existence of such an anomalous diffusive region is due to the resonance-induced wave focusing effect that makes the directional randomization difficult during the scattering process. The diffusion approximation is valid when $L>L_{c}$. The value of $L_{c}$ is about ten times the average extrapolation length, i.e., $L \approx 10 z_{e}$, where $z_{e}$ is a fast increasing function of the amount of internal reflection.

\section{ACKNOWLEDGMENT}

This work was supported by Hong Kong RGC, Grant No. HKUST 6163/01P. 
[1] See, for example, Scattering and Localization of Classical Waves in Random Media, edited by P. Sheng (World Scientific, Singapore, 1990); P. Sheng, Introduction to Wave Scattering, Localization and Mesoscopic Phenomena (Academic Press, New York, 1995); M.C.W. van Rossum and T.M. Nieuwenhuizen, Rev. Mod. Phys. 71, 313 (1999).

[2] Y. Kuga and A. Ishimaru, J. Opt. Soc. Am. A 1, 831 (1984); M.P. Van Albada and A. Lagendijk, Phys. Rev. Lett. 55, 2692 (1985); P.E. Wolf and G. Maret, ibid. 55, 2696 (1985); E. Akkermans, P.E. Wolf, and R. Maynard, ibid. 56, 1471 (1986); M. Kaveh, M. Rosenbluh, I. Edrei, and I. Freund, ibid. 57, 2049 (1986).

[3] J.H. Li, A.A. Lisyansky, T.D. Cheung, D. Livdan and A.Z. Genack, Europhys. Lett. 22, 675 (1993).

[4] J.H. Page, H.P. Schriemer, A.E. Bailey, and D.A. Weitz, Phys. Rev. E 52, 3106 (1995).

[5] E. Wolf, Phys. Rev. Lett. 56, 1370 (1986); B. Shapiro, ibid. 57, 2168 (1986); M. Stephen and G. Cwilich, ibid. 59, 285 (1987); S. Feng, C. Kane, P.A. Lee, and A.D. Stone, ibid. 61, 834 (1988); I. Freund, M. Rosenbluh, and S. Feng, ibid. 61, 2328 (1988); N. Garcia and A.Z. Genack, ibid. 63, 1678 (1989); R. Berkovits, M. Kaveh, and S. Feng, Phys. Rev. B 40, 737 (1989); M.P. van Albada, J.F. de Boer, and A. Lagendijk, Phys. Rev. Lett. 64, 2787 (1990).

[6] D.A. Weitz, D.J. Pine, P.N. Pusey, and R.J.A. Tough, Phys. Rev. Lett. 63, 1747 (1989); I. Freund, M. Kaveh, and M. Rosenbluh, ibid. 60, 1130 (1988).

[7] R.H.J. Kop, P. de Vries, R. Sprik, and A. Lagendijk, Phys. Rev. Lett. 79, 4369 (1997).

[8] K.M. Yoo, F. Liu, and R.R. Alfano, Phys. Rev. Lett. 64, 2647 (1990).

[9] K.M. Yoo and R.R. Alfano, Opt. Lett. 15, 320 (1990).
[10] F.C. MacKintosh and S. John, Phys. Rev. B 40, 2383 (1989).

[11] P.-A. Lemieux, M.U. Vera, and D.J. Durian, Phys. Rev. E 57, 4498 (1998).

[12] Z.Q. Zhang, I.P. Jones, H.P. Schriemer, J.H. Page, D.A. Weitz, and P. Sheng, Phys. Rev. E 60, 4843 (1999); Z.Q. Zhang and P. Sheng, in Photonic Band Gap Materials, Vol. 315 of NATO Advanced Study Institute, Series E: Applied Sciences, edited by C.M. Soukoulis (Kluwer Academic Publishers, Dordrecht, Boston, 1996).

[13] A.A. Chabanov and A.Z. Genack, Phys. Rev. E 56, R1338 (1997).

[14] A. Garcia-Martin, J.J. Saenz, and M. Nieto-Vesperinas, Phys. Rev. Lett. 84, 3578 (2000).

[15] A. Lagendijk, R. Vreeker, and P.D. Vries, Phys. Lett. A 136, 81 (1989).

[16] J.X. Zhu, D.J. Pine, and D.A. Weitz, Phys. Rev. A 44, 3948 (1991).

[17] I. Freund and R. Berkovits, Phys. Rev. B 41, 496 (1990).

[18] I. Freund, Phys. Rev. A 45, 8854 (1992).

[19] A.A. Lisyansky and D. Livdan, Phys. Lett. A 170, 53 (1992).

[20] Th.M. Nieuwenhuizen and J.M. Luck, Phys. Rev. E 48, 569 (1993).

[21] H.A. Fertig and S.D. Sarma, Phys. Rev. B 40, 7410 (1989).

[22] M.B. van der Mark, M.P. van Albada, and A. Lagendijk, Phys. Rev. B 37, 3575 (1988).

[23] K.E. Atkinson, A Survey of Numerical Methods for the Solution of Fredholm Integral Equations of the Second Kind (Society for Industrial and Applied Mathematics, Philadelphia, 1976).

[24] A. Ishimaru, Wave Propagation and Scattering in Random Media (Academic, New York, NY, 1978).

[25] J.G. Rivas, R. Sprik, A. Lagendijk, L.D. Noordam, and C.W. Rella, Phys. Rev. E 63, 046613 (2001). 